\title{
Grants proposals and animal use protocols: Is the IACUC playing by the rules?
}

Dr. Harry Markowitz, an established cancer investigator at Great Eastern University, submitted a research grant to the National Institutes of Health (NIH). The NIH gave the grant a priority score of 130, which would probably result in the project being funded. Markowitz then submitted a protocol application to the IACUC. After deliberation the Committee requested a change in the number of animals to be approved, asking Markowitz to perform pilot studies to see if some of the proposed gene therapy experiments would actually be necessary. If the pilot studies showed any positive results he could then always amend his protocol to request more animals. Markowitz readily agreed to this condition. The Committee also requested some significant changes in the care of animals before and after the procedure in one of his planned experiments, to which Markowitz also agreed. Once all concerns were met, the IACUC approved the protocol.
Janis Hart was a graduate student in Markowitz's laboratory. She and Markowitz never really 'clicked', and eventually Hart began to complain that Markowitz was harassing her. Perhaps there should have been a resolution to the problem at this point, but this did not happen, and eventually Hart contacted the Office of Laboratory Animal Welfare (OLAW) and the federal Office of Research Integrity (ORI), alleging scientific fraud (fabrication of data) and animal abuse. She provided what she considered to be adequate proof of both charges. Both federal organizations immediately contacted the appropriate officials at Great Eastern and requested an internal investigation. ORI and OLAW finally cleared Markowitz of the specific allegations, but secondary issues arose with OLAW. For example, according to Great Eastern's own documentation, it was apparent that the IACUC's letter of approval verification to OLAW did not include the IACUC's approved changes to Markowitz's protocol. Furthermore, the Great Eastern IACUC never compared the protocol application with the grant application, and there were animal studies in the grant that were not described in the IACUC protocol, and conversely, animal studies on the IACUC protocol that were not in the grant. OLAW asked for an explanation. Great Eastern responded that Markowitz decided not to perform some of the mouse studies described in the grant application, and thus he did not include them on the IACUC application. In their place he substituted related rat studies, which the IACUC reviewed in full. Neither Markowitz nor the IACUC considered these changes to be significant because of the inherent flexibility allowed under the NIH's modular grant format. Furthermore, the IACUC was under the impression that so long as it approved the final version of the protocol, there was no need to document to NIH/OLAW any of the changes made as a consequence of the review process.

Was Great Eastern playing within the rules or just playing with the rules?

\section{RESPONSE}

\section{'Instant replay' required}

\section{H. Edwin Kennah, PhD \& \\ Paul H. Bramson, DVM, MS}

From the foregoing scenario it is impossible to determine if Great Eastern is playing with the rules or just 'dropped the ball' on this play. However, it appears that OLAW is correct in reprimanding Great Eastern for not conforming to the rules as stated in section IV.C of the Public Health Service Policy on Humane Care and Use of Laboratory Animals (PHS Policy). This policy requires the review and approval by the IACUC of all components of grant applications and contract proposals that relate to the care and use of animals. In addition, the signature of the authorized Institutional Official on the 398 grant application form certifies that the IACUC will or has reviewed and approved all animal-related activities in the grant application. It seems that the Great Eastern IACUC needs to develop a procedure for ensuring compliance with the requirements for review of a grant application's animal care and use procedures. The IACUC only reviewed the protocol review form on which Markowitz provided information about his proposed use of animals. A procedure for the direct comparison of the information on the grant application form with the IACUC protocol review form may have kept Great Eastern out of the OLAW penalty box.
The second penalty called by OLAW was for failure to report changes made in the grant proposal. Markowitz decided to substitute some mouse studies described in the grant proposal with related studies on rats. The IACUC approved these changes as part of their review of Markowitz's protocol review form, but did not understand the need to report them to NIH/OLAW. Because the IACUC's review was performed after the grant application's scientific review at NIH, PHS Policy IV.D.2 requires that any changes in the proposal be forwarded to the $\mathrm{NIH}$ as part of the delayed-approval notification process. Great Eastern's contention that the changes are not significant will not stand up to review, because OLAW guidance ${ }^{1}$ states that a change in species is significant. Therefore, the Great Eastern challenge of the 